\title{
Publizistik und Sozialpädagogik
}

\author{
von Peter Rech
}

Es gibt einen sehr ursprünglichen Zusammenhang von Pädagogik und Publizistik. Schleiermacher hat ihn erstmals ausgesprochen, als er die Politik mit der Pädagogik "koordinierte“.1 In diesem Zusammenhang verstand er Politik dialogisch als kommunikatorisches Verhältnis der gegenseitigen Einwirkung gleichberechtigter Bürger eines Gemeinwesens oder Staates. Pädagogik verstand sich dementsprechend als ungleichseitiges, nämlich einseitiges Einwirkungsverhältnis der älteren Generation auf die jüngere, d.h. erweitert gesehen: der selbständigeren Generation auf die noch hilfsbedürftige. Beide nur im Gesellschaftlichen $\mathrm{zu}$ fassende Begegnungsverhältnisse trugen Sorge um die Weckung und Pflege des Bewußtseins des einzelnen, das sich multipliziert in der Vernünftigkeit (sittlicher Standpunkt) der Gesellschaft konkretisierte und sich besonders deutlich im sozialen Prozeß der Mitteilung artikulierte. Dabei wurde das implizierte dauernde Reformbestreben zur Verhütung von zerstörerischen Revolutionen als von oben eingeleitet und aufrechterhalten betrachtet: Die Meinungsträger sollten aus hohem sittlichem Allgemeininteresse handeln und nicht aus dem „sinnlichen Kalkül“ (egoistische Eigeninteressen). ${ }^{2}$

Bei Schleiermacher gehören Politik als Kommunikationsverhältnis und gesellschaftlich eingestellte Pädagogik so zusammen wie Theorie und Praxis aufeinander angewiesen sind, insofern beide Wissenschaften Anwendungsfälle der ein geregeltes Zusammenleben ermöglichenden, als Kulturanthropologie verstandenen Ethik sind. Dieses Verständnis entspricht dem (leider nicht lange anhaltenden) reformerischen Politik-Verständnis in Preußen Anfang des 19. Jahrhunderts ${ }^{3}$ : Menschenbildung war eng mit dem Leben im Staate verbunden. Die Gefahr eines sich verselbständigenden bürokratischen Geschäftsgeistes war damals schon erkannt worden. Die „wissenschaftliche Deputation" hatte neben ihrer allgemeinen staatskontrollierenden (!) Funktion zur Aufgabe, die entsprechende Verwaltung im Sinne des geistig-kulturellen Fortschritts zu dynamisieren.

Die Konstitution der Praxis in der Schleiermacherschen „Theorie der Erziehung“4 deutet - das sei hier nur kurz angesprochen - auf das emanzipatorische Anliegen hin, das heute die kritische Theorie anhand des „erkenntnisleitenden Interesses“ um der Ermöglichung des gesellschaftlichen Fortschritts willen verfolgt. ${ }^{5}$

Die Aufgabe der politischen Meinungsbildung ist zunehmend dem Publizisten zugefallen, während dem Politiker die Organisation, d. h. die Bewältigung der Bürokratie einer Massengesellschaft obliegt. Aus der Konstellation der Industriegesellschaft haben sich Strukturen herausgebildet, die das System der Organisation selbständig gemacht, Praxis und Theorie „arbeitsteilig“ getrennt und das Zusammenleben und die Zusammenschau dem einzelnen Bürger erschwert haben. Aus diesem Zusammenhang bildeten sich gleichermaßen Sozialpädagogik und Publizistik heraus. ${ }^{6}$

Peter Rech hat sich nach beruflicher Tätigkeit als Lehrer dem Studium der Pädagogik, Publizistik und Psychologie an der Universität Münster zugewandt. 
Wie Sozialpädagogik deshalb als "Bestandteil desjenigen pädagogischen Systems“ ausgelegt wird, "das durch die industrielle Gesellschaft hervorgebracht wurde" , so ist Publizistik entsprechend als Bestandteil des gleichseitigen Kommunikationssystems der Industriegesellschaft aufzufassen; negativ ausgedrückt: „Je unentwickelter das Gemeinschaftsleben eines Volkes, desto unentbehrlicher seine Durchleuchtung und Förderung durch die publizistische Forschung." 8

Es muß an dieser Stelle darauf hingewiesen werden, daß Sozialpädagogik keineswegs als eigenständige Wissenschaft aufgefaßt wird, sondern ähnlich wie die "soziologische Pädagogik" nicht nur Teil der Wissenschaft Pädagogik ist, sondern Pädagogik selbst in einer besonderen Sicht- und darüberhinaus Handlungsweise. ${ }^{8}$ Dabei hat sie - um es noch einmal zu wiederholen - ihre historische Begründung in einer den Ansprüchen der Industriegesellschaft nicht mehr genügenden individuell eingestellten Pädagogik genauso wie die öffentlich-politisch wirksame Publizistik gegenüber einer von wenigen normierten Politik in der ständischen Gesellschaft.

Die intuitive sozialphilosophische Diskussion (die „soziale Frage“), die das Geistesleben des 19. Jahrhunderts noch sehr stark bestimmte und die Soziologie als empirisch kontrollierende Wissenschaft überhaupt erst anregen konnte, hat die bis dahin meist individualistisch gefärbte Pädagogik um bestimmte Sichtweisen bereichert.10 Sie griff schließlich - als soziologische Diskussion - auf den gesamten Bereich der Erziehung über und vertrat bald nur noch eine soziologische Sinngebung der Erziehung, nicht ihre eigentlich pädagogische. Solche Auffassungen konnten die Erziehungswirklichkeit nicht im Ganzen in den Griff bekommen und Erziehung nur als „Eingliederung und Typisierung " begreifen. ${ }^{11}$ Mit dieser soziologischen „Standpunkt-Pädagogik "12 ist jedoch das Wesentliche der Erziehung verundeutlicht, daß nämlich dem Erzieher die Verantwortung von keiner Gemeinschaft abgenommen werden kann. ${ }^{13}$

Aloys Fischer, der den erzieherischen Einfluß der Gesellschaft ganz und gar anerkennt, sieht das pädagogische, also auch das sozialpädagogische Geschehen jeweils „in der persönlichen Begegnung zwischen zwei Menschen " realisiert, „in welcher der Wissende die geistige Stellvertretung des noch Unmündigen übernimmt. " ${ }_{14}$

Der Publizist und das Publikum stehen als Kommunikator und Rezipient in einem entsprechend dialogischen, aber gleichseitigen Verhältnis: „Publizistik ist Zwiegespräch. ${ }{ }^{15}$

In der spätkapitalistischen Gesellschaft entspricht die Immanenz von Kollektiveigenschaften, die man gerne den Mitgliedern einer sich nivellierenden Masse zuschreiben möchte, der Eminenz der nach dem "sinnlichen Kalkül" handelnden und meinungsbildenden Kräfte, mit denen einzelne mächtige (meist besitzende) Menschen ihren Einfluß sichern. Die Anonymität der entfremdeten Massenmenschen wird ebenso wie jede andere Unmündigkeit des Menschen als selbstverschuldet angesehen werden müssen.

„Aufklärung ist der Ausgang des Menschen aus seiner selbstverschuldeten Unmündigkeit. ${ }^{16}$... Zu dieser Aufklärung aber wird nichts erfordert als Freibeit, und zwar die unschädlichste unter allem, was nur Freiheit heißen mag, nämlich die: „von seiner Vernunft in allen Stücken öffentlichen Gebrauch zu machen." ${ }^{17}$

Das nicht "aufgeklärte" Publikum wird durch die Verpflichtung zur allgemeinen Publizität im Gegensatz zu den Absichten einer nicht öffentlichen "absoluten" Regierung verunsichert werden müssen: „In einer dynamișchen Gesellschaft gehören 
Emanzipations-Erscheinungen zum Gesamtprozeß. Sie können damit keinesfalls als Auflösungserscheinungen verstanden werden, obwohl sie von einem blinden Konservatismus in diesem Sinne empfunden werden." 18

Die Rolle der jungen Politikwissenschaft kann die der Publizistikwissenschaft nicht ersetzen. Politische Wissenschaft wird sich an der politischen Organisation messen müssen, welche in einer Massengesellschaft des publizistischen Handelns bedarf. ${ }^{19}$

Während die praktisch arbeitenden Publizisten ihrer sittlichen Verantwortung aus dem formalen Anspruch der öffentlichen Aufgabe oft nicht genügen können, weil sie als Lohnabhängige ihren Arbeitsbereich auch, vielleicht sogar zunehmend, von Verdienstmöglichkeiten bestimmen lassen müssen, befinden sich die praktisch arbeitenden Sozialpädagogen heute entgegen ihrer "öffentlichen Aufgabe" in einer gewissen Abhängigkeit von institutionalisierten, wiederum wirtschaftlich geprägten Vorstellungen von Jugendarbeit und Erwachsenenbildung. Die Vermarktung der Presse ist wie die Institutionalisierung sozialpädagogischer Einrichtungen auf Entwicklungsvorgänge in der Massengesellschaft zurückzuführen. Der Grund bei der Presse und den anderen Massenmedien liegt in der komplizierten Technisierung, die erst bestimmte Auflagenhöhen und entsprechende Ausstrahlungsbereiche ermöglichte und in der „immanenten Entdeckung" werbepsychologisch wirksamer Kommunikationsmethoden und Kommunikationstechniken, die die kritische Auseinandersetzung mit den Konsequenzen eines öffentlichen Zustandes mehr oder weniger bewußt vernachlässigten. Die Institutionalisierung der sozialpädagogischen Einrichtungen zur Erhaltung der seelischen Gesundheit in der Gesellschaft hat eine unter Umständen theorie-entgegengesetzte Gliederung nach sog. „dahinterstehenden“ (politischen, kirchlichen, wirtschaftlichen) Interessen der Trägerverbände mit sich gebracht.

Publizistik und Sozialpädagogik sind beide „engagierte" und auf positive Veränderung der Gesellschaft angelegte Wissenschaften, während die Politik in einer arbeitsteiligen Massengesellschaft immer mehr als Organisations- und Erhaltungs- und weniger als Kontrollfaktor ${ }^{20}$ erachtet werden muß. Das konkrete Problem beider Wissenschaften liegt heute in der noch völlig unbeantworteten Frage nach der Segmentierung der Gesellschaft im Interesse des einzelnen, der diese zu überblicken nicht mehr in der Lage ist. Beide Wissenschaften beleuchten die typischen Formen der Verwahrlosung im Industriezeitalter, nämlich auf der einen Seite die Verwahrlosung des öffentlichen Zustandes überhaupt und auf der anderen Seite die seelische Verwahrlosung des einzelnen oder verschiedener Gruppen. Beide Wissenschaften beschäftigen sich heute weniger mit methodischen Fragen als mit der Problematisierung eines gesellschaftlichen Bewußtseins. Sieht Hans Böttcher die Problematik des Verhältnisses von Pädagogik und Publizistik noch in der „Divergenz publizistischer und pädagogischer Intentionen "21, so wird man doch gegensätzlich festhalten müssen, daß Sozialpädagogik in der Besinnung auf gesellschaftliche Aufgaben nicht nur im didaktischen, sondern gerade auch im intentional-normativen Bereich mit der Publizistik übereinstimmt. Das Verhältnis von Pädagogik und Publizistik ist weniger subjektiv durch die Antithese methodische Konvergenz und intentionale Divergenz als objektiv durch den schon von Schleiermacher und später Fischer dargestellten Gegensatz von der Gleichberechtigung der Generationen auf der einen und der Stellvertretung der älteren Generation auf der anderen Seite gekennzeichnet.

Das pädagogische Prinzip der Stellvertretung erscheint vielleicht manchem innerhalb der Sozialpädagogik, die es oft genug mit Erwachsenen zu tun hat, als unangebracht. Pädagogik in ihrer letzten existentiellen Konsequenz ist nicht Stellvertretung um 
ihrer selbst willen; als Entsprechung menschlicher Unvollkommenheit will sie zum selbstverantworteten Handeln führen. In diesem Sinne deutet Otto F. Bollnow die unstetigen Formen der Erziehung, z. B. die Beratung, die allerdings „aus besserer Sachkenntnis des Ratenden die erforderliche Einsicht in die Situation (entwickelt) ${ }^{{ }_{\mathbf{2 2} 2}}$.

So zeigt sich gerade an der institutionalisierten Beratung, der klassischen sozialpädagogischen Einrichtung der Erziehungsberatung, die Notwendigkeit ihrer pädagogischen Begründung, weil sie, wenn sie nur psychologisch begründet würde, zum bloßen Experimentierfeld berechtigterweise ausarten könnte; in einer soziologischen Begründung könnte sie nur Bedeutung als Material zur Analyse von bestimmten Interaktionen haben, und wenn sie ihre Begründung nur in einer Ethik finden könnte, würde sie mit Sicherheit zum Spielball von Ideologien werden und darüberhinaus ihre eigentliche, jeweils gegenwärtige stellvertretende Verantwortung vergessen.

Mit dieser Einschränkung soll nicht gesagt werden, daß Sozialpädagogik in Publizistik eingehen kann oder umgekehrt. Obwohl - wie unten noch dargestellt wird in der Publizistik pädagogische Dimensionen sichtbar werden, muß eine primär pädagogische Publizistik gefährlich sein, weil sie - per definitionem - eine Stellvertreter-Rolle übernimmt, die ihr unter erwachsenen Menschen nicht zukommen darf. Publizistische Sozialpädagogik könnte nur dort ein Teilgebiet der Pädagogik sein, wo sich diese um methodische Maßnahmen bemüht.

Die Aufgabe der Pädagogik liegt zu einem Teil in der Ermöglichung, die der Sozialpädagogik in der Sicherung publizistischer Prozesse. Dabei kann schon die Schulpädagogik publizistische Methoden vorwegnehmen, z. B. schulimmanente Offentlichkeitsarbeit: SMV und Schülerzeitung; darüberhinaus Kontaktnahme zur Publizistik überhaupt: Teilnahme an den Massenmedien in der Schule; schließlich Ubernahme publizistischer Methoden: z. B. Leserbriefaktionen, Gestaltung eigener Programme mit Hilfe von Video-Recordern usw.

Wenn „die Publizistik ... ihrer Natur nach nicht dazu geschaffen [ist], ein konstruktives zusammenhängendes Weltbild zu vermitteln", so ist zumindest die Sozialpädagogik als überschulische Pädagogik ebenfalls dazu nicht in der Lage, sondern sie soll vielmehr dem Menschen in einem unzusammenhängenden Weltbild Lebensmöglichkeiten aufzeigen. ${ }^{23}$

„Einen Publizisten können bei seiner Arbeit kulturelle oder politische, pädagogische oder missionarische Absichten bewegen. "24 Entsprechend bezeichnen mit den gleichen propagandistischen Begründungen verschiedenste weltanschauliche Richtungen jeweils ihre Pädagogik als die einzig wahre. Gegenüber diesen an Umstände von Weltanschauung gebundenen Pädagogiken existiert jedoch endlich immer „die Wissenschaft Pädagogik" ${ }^{25}$, nämlich die "Theorie des sinnhaften Lebens", das in Isolation nicht möglich erscheint, ,in Anwendung auf die besondere pädagogische Situation ${ }^{\alpha 28}$, in diesem Fall auf die sozialpädagogische. In der gleichen Weise gibt es die Wissenschaft Publizistik, die sich als Kommunikationswissenschaft dem Anspruch der Offentlichkeit verpflichtet weiß.

Die grundsätzliche Einsicht z. B., daß jede pädagogische Verfahrensweise sich „aus der Plastizität des empirischen Menschenmaterials und der Dynamik der Beeinflussungsmittel ${ }^{{ }^{27}}$ ergibt, gilt in formaler Hinsicht genauso der Publizistik, wobei nicht vergessen wird, daß die Pädagogik aus ihrer stellvertretenden Stellung heraus 
tatsächlich eine größere „Plastizität" antrifft und daher - überraschenderweise? in ihrer möglichen negativen Wirkung mehr manipulieren kann als jede „spätere“ Publizistik.

Sozialpädagogik wie Publizistik befinden sich in dem grundsätzlichen fruchtbaren Spannungsverhältnis von Theorie und Praxis. Nicht nur in der Publizistik „ist unverkennbar, daß die Praxis derartig komplex rationale Ansprüche stellt, daß sie verstärkter Rationalisierung bedarf“". ${ }^{8}$

Franz Dröge differenziert publizistisches Arbeiten in einem Handlungsschema ${ }^{29}$, das das Ineinandergreifen von Theorie und Praxis auf der einen Seite wie auch die theoretische Notwendigkeit der Analyse auf der anderen Seite dokumentiert. Dieses Modell läßt sich ebenso auf die Sozialpädagogik übertragen: Beide Wissenschaften zeichnen sich durch das Interesse an der Gesellschaft aus, wobei verselbständigte Ziele und "wertneutrale“ Materialien in beiden Fällen nicht zur - im antizipatorischen Sinne - notwendigen Theoriebildung führen. Beide Wissenschaften finden in der Soziologie die der Theoriebildung und der Praxis vorausgehende "Nachhinein-Wissenschaft " zur Analyse der Gesellschaft.

Weder die eine noch die andere Wissenschaft ist vorgängig. Publizistik übernimmt eine sozialpädagogische Aufgabe, insoweit sie Lebensmöglichkeiten erleichtert. Publizistik arbeitet ziel-immanent, insoweit sie sozialkommunikatorische Prozesse untersucht und ermöglicht. Das Problem von Gemeinschaft und Gesellschaft als zu bewertende Lebensformen ist ein altes Anliegen der Sozialpädagogik (nicht der Soziologie; dort lediglich Begriffsdiskussion) und wird dort weiter diskutiert unter dem Aspekt der Lebensmöglichkeiten in der Massengesellschaft und des Problems der Sozialsituation.

Neben dieser Koordination hinsichtlich der Kritik der Unübersichtlichkeit kommunikatorischer Prozesse in der Massengesellschaft überschneiden sich beide Wissenschaftsbereiche - wie gesagt - in den Fragen kommunikativer Methoden und stehen in einem Funktionszusammenhang in den Fragen der Beeinflußbarkeit und den Folgen der Beeinflussung einzelner Menschen durch marktbestimmte, psychologisch-effektiv und nicht pädagogisch- bzw. publizistisch-intentional methodisierte Massenmedien und der sozialpsychologischen Publikums- und Wirkungsforschung.

Aus diesem Zusammenhang der Wissenschaftsbereiche Sozialpädagogik, Politikwissenschaft und Publizistik und deren "Humanpraktika" soziale Erziehung (Vorbereitung auf gesellschaftliches Zusammenleben), Politik (Organisation und Lenkung der Gesellschaft) und Journalistik (Information über gesellschaftliche Ereignisse und Kommentierung gesellschaftlicher Ereignisse und Unterhaltung der Gesellschaft) ergibt sich die pädagogische, d.h. die Praeceptor-Funktion der Journalistik als praktisch arbeitende Publizistik. Den zentralen Bereich stellt die Politik dar, die innerhalb einer demokratischen Staatsform gesellschaftliche Interessen-Spannungen auszugleichen versucht. Pädagogik kann nicht nur Anpassung an die Gesellschaft bedeuten, weil sie den immer neu zu ermittelnden Ansprüchen der jüngeren Generation zu entsprechen hat. Pädagogik ist der Politik im antizipatorischen Sinne insofern vorgeordnet, als sie in eine zukünftige Gesellschaft hineinerzieht.

Publizistik kann sich entsprechend nicht nur als Reproduktion der Gesellschaft auffassen, weil sie die Ansprüche der Gesellschaft nach Offentlichkeit immer wieder neu zu überprüfen hat. So gesehen ist Publizistik der Politik im emanzipatorischen Sinne nachgeordnet. Der Lehrer erzieht für die Gesellschaft, der Journalist infor- 
miert über die Gesellschaft. Der Lehrer wird bei seiner Aufgabe ohne die journalistische Tätigkeit der Information nicht auskommen, wie der Journalist ohne pädagogische Artikulation nicht auskommen wird, wenn er Widersprüche zwischen gesellschaftlichem Anspruch (z. B. Grundgesetz) und gesellschaftlicher Realität aufdeckt. Dabei wird das publizistische Gewissen nicht objektivierbar sein, sondern von der "präzeptorischen“ Interpretation aufgrund weltanschaulicher Gebundenheit, $\mathrm{zu}$ der freiheitliche gesellschaftliche Ordnungen Raum geben müssen, abhängen, weshalb die pädagogische Artikulation bei einem Journalisten deutlicher zum Vorschein kommen kann als bei einem anderen.

\section{Anmerkungen:}

1. Vgl. Friedrich Ernst Daniel Schleiermacher: Ausgewählte pädagogische Schriften. Besorgt von Ernst Lichtenstein, Paderborn 2/1964, bes. S. 42 (Vorlesungen aus dem Jahre 1826).

2. Vgl. Der problematische Mensch als Gegebenheit, in: Horst Friebel: Die Bedeutung des Bösen für die Entwicklung der Pädagogik Schleiermachers, Ratingen 1961, bes. S. $94 \mathrm{ff}$.

3. Vgl. dazu Ernst Lichtenstein: Schleiermachers Pädagogik. O. F. Bollnow zum 65. Geburtstag, in: "Neue Zeitschrift für Systematische Theologie und Religionsphilosophie“, 10. Bd. 1968 , S. 345 .

4. Gemeint sind die a.a.O. von Ernst Lichtenstein so überschriebenen Vorlesungen aus dem Jahre 1826.

5. Vgl. Jürgen Habermas: Erkenntnis und Interesse, in: ${ }_{n}$ Merkur ${ }^{\alpha}, 1965$, S. $1139 \mathrm{ff}$ und Herwig Blankertz: Pädagogische Theorie und empirische Forschung, in: „Zur Bedeutung der Empirie für die Pädagogik als Wissenschaft ${ }^{\alpha}$, Neue Folge der Ergänzungshefte zur „Vierteljahrsschrift für wissenschaftliche Pädagogik“, Heft 5, bes. S. 74 f.

6. Ernst Bornemann: Die Bedeutung der Sozialpädagogik in der industriellen Gesellschaft, in: „Soziale Welt“, Band 11, S. $213 \mathrm{ff}$. Walter Hagemann: Grundzüge der Publizistik. Neu herausgegeben von Henk Prakke unter Mitarbeit von Winfried B. Lerg und Michael Schmolke, Münster 21966, S. 211.

7. K. Mollenhauer: Einführung in die Sozialpädagogik, Weinheim 1964, S. 19, zit. nach Lutz Rössner: Sozialpädagogik, in: Josef Speck und Gerhard Wehle (Hrsg.): Handbuch pädagogischer Grundbegriffe, München 1970, Bd. II, S. 467.

8. Hagemann, a.a.O., S. 276.

9. Vgl. hier und im folgenden: Aloys Fischer: Soziologische Pädagogik (1932), in: Karl Kreitmar (Hrsg.): Aloys Fischer. Leben und Werk, Bd. 3/4: Gesammelte Abhandlungen zu Soziologie, Sozialpädagogik und Sozialpsychologie, München 1954, S. 159.

10. Vgl. ebd., S. $162 \mathrm{f}$.

11. Vgl. Ernst Krieck: Erziehungsphilosophie, München 1930, S. 69, zit. nach Hermann Röhrs: Die Pädagogik Aloys Fischers. Versuch einer systematischen Darstellung seines wissenschaftlichen Gesamtwerkes, Heidelberg 21967, S. 83.

12. Fischer, a.a.O., S. 162.

13. Vgl. auch Röhrs, a.a.O., S. 83.

14. Ebd., S. 83.

15. Hendricus Johannes Prakke: Sozialerziehung und Massenpublizistik, in: Ernst Bornemann und Gustav von Mann-Tiechler (Hrsg.): Handbuch der Sozialerziehung, Bd. II, Praxis der Sozialerziehung im geordneten sozialen Feld, Freiburg/Br. 1964, S. 266. Ders.: Alle Publizistik ist Zwiegespräch, in: „Publizistik“, Jg. 5/1960, S. $208 \mathrm{ff}$.

16. Immanuel Kant: Beantwortung der Frage: Was ist Aufklärung? (1874), in: Wilhelm Weischedel (Hrsg.): Immanuel Kant. Werke in zehn Bänden, Bd. 9 und 10: Schriften zur Anthropologie, Geschichtsphilosophie, Politik und Pädagogik, Darmstadt 1970, S. 53.

17. Ebd., S. 55.

18. Vgl. Heinrich Rombach: Emanzipation, in: Lexikon der Pädagogik, Neue Ausgabe, Freiburg/Basel/Wien 1970, Bd. I.

19. Vgl. Marian Heitger: Pädagogik als Wissenschaft und ihre gesellschaftliche Verantwortung, in: „Viertel jahrsschrift für Wissenschafts-Pädagogik“, Heft 3/1968 (Ehrengabe an Kurt Haase zur Vollendung seines 70. Lebensjahres), S. 185. 
20. Vgl. die pädagogische Dimension dieser Entwicklung und ihre Problematik in: Klaus Wallraven und Eckart Dietrich: Politische Pädagogik. Aus dem Vokabular der Anpassung, München 1970 (Reihe Hanser), bes. folgende Schlüsselbegriffe: Parlamentarismus (S. $72 \mathrm{ff}$ ), Politik (S. $89 \mathrm{ff}$ ), Verantwortung (S. $94 \mathrm{ff}$ ).

21. Hans Böttcher: Der Jugendliche im Wirkungsfeld publizistischer und wirtschaftlicher Kräfte, in: Ernst Bornemann und Gustav Mann-Tiechler (Hrsg.), a.a.O., S. 274.

22. Otto Friedrich Bollnow: Existenzphilosophie und Pädagogik, Stuttgart 1965, S. 81.

23. Böttcher, a.a.O., S. 273.

24. Prakke, a.a.O., S. 267.

25. Fischer, a.a.O., S. 161.

26. Heinrich Döpp-Vorwald: Erziehender Unterricht und menschliche Existenz (1932), Darmstadt 1968, S. 9.

27. Fischer, a.a.O., S. 161.

28. Franz W. Dröge: Regel und Regelung. Ansätze der Publizistikwissenschaft, in: „Publizistik“, Jg. 11/1966, S. 150.

29. Ebd.

\section{S U M M A R Y}

The wide-spread assumption that pedagogy and communications contradict each other as far as their intention or goals are concerned, is repealed by confrontation of social pedagogy and communications. This shows, that the assumed relation in social communications (communicatio socialis) between pedagogy and communications, has already been stated by Schleiermacher through the coordination of pedagogy with politics. In claiming to be society oriented, social pedagogy and communications equally try to locate and abolish the marks of degeneration in the industrial era - „degeneration “ of the public situation and the moral degeneration of the individual or of groups. Both sciences are, in the trend of commercialisation of the press and the government censorship of social pedagogy bodies' interests exposed, and have, while forming their theories, to concentrate again more on their goals rather than on their methods. Both sciences develop, out of the critical relationship of theory and practice, models to overcome the obscurity of communications processes in society.

\section{RES UMEN}

La opinión, muy extendida, de que la Pedagogía y el Periodismo se contradicen en el sector intencional desaparece cuando se confronta la Pedagogía Social con el Periodismo. En esta confrontación aparece que la supuesta relación entre la Pedagogía y el Periodismo, en el campo de las Comunicaciones Sociales, fue definida realmente por Schleiermacher mediante la coordinación de la Pedagogía con la Política. Con una pretensión social, tanto la Pedagogía social como el Periodismo tratan de descubrir y eliminar las características del abandono de la época industrial - „abandono ${ }^{\alpha}$ del estado público y abandono espiritual del individuo o grupos. Ambas ciencias, bajo la corriente de comercialización de las empresas periodísticas y bajo la inspección estatal de las instituciones social-pedagógicas, dependen de ciertos intereses $y$, en la formación de sus teorías, deben concentrarse no tanto en sus métodos cuanto en el establecimiento de metas. De esta relación de tensión entre la teoría y la práctica, ambas ciencias desarrollan modelos para vencer los intrincados procesos de comunicación en la sociedad de masas.

Además de esta relación de coordinación en lo intencional, ambas ciencias se estorban en cuestiones metódicas y se hallan en una relación funcional en las cuestiones de la influencia y capacidad de dejarse influenciar de algunos individuos y en las consecuencias para la sociedad.

De estas relaciones resulta concretamente la función docente del periodista. 